\title{
Tiempos y barreras de acceso para procedimientos quirúrgicos electivos en la población de Viracachá (Boyacá, Colombia), durante el periodo 2018-2019
}

\author{
Cristian Camilo Becerra Gómez¹ (D) , Javier Martínez Torres² (D)
}

\begin{abstract}
RESUMEN
Introducción: se ha descrito ampliamente que el acceso a procedimientos quirúrgicos oportunos es esencial para la equidad de atención en salud, bienestar, disminución de la morbimortalidad y aumento del desarrollo económico. Las listas de espera de cirugías electivas son un problema para los sistemas de salud, debido al desequilibrio existente entre la demanda de pacientes y la capacidad para proveer oportunamente dichos procedimientos, principalmente en áreas rurales, donde están mucho más marcadas las restricciones financieras y geográficas, factores administrativos, creencias culturales y pobre educación.

Objetivo: determinar los tiempos de espera y las barreras para procedimientos quirúrgicos electivos en pacientes atendidos en la ESE Centro de Salud de Viracachá (Boyacá, Colombia), durante 2018 y 2019.

Metodología: estudio de seguimiento a una cohorte, con descripción sociodemográfica, clasificación de causas de demora de cirugía electiva y análisis por tipos de variables, mediante tasas persona-tiempo.

Resultados: el 48,1\% de los pacientes tuvieron el procedimiento quirúrgico electivo; en general, se tuvo un tiempo de espera de 9,4 meses ( $D E=3,4$ ) y una tasa de operaciones por cada 100 meses de 5,13 (IC 95\%: 2,85-8,56). Los grupos con mayores tasas de operaciones por cada 100 meses fueron pacientes que pertenecían al estrato 1, los que percibieron una causa administrativa asociada con demora de la cirugía electiva y aquellos que tuvieron una cirugía ortopédica. Conclusión: las largas listas de espera para las cirugías electivas pueden tener un componente multifactorial, el cual es necesario identificar para plantear políticas estratégicas que permitan su acceso oportuno, principalmente en áreas rurales.
\end{abstract}

Palabras clave: procedimientos quirúrgicos; servicios ambulatorios; barreras de acceso a los servicios de salud; servicios de salud; población rural; Colombia.

\footnotetext{
${ }^{1}$ E.S.E. Centro de Salud Viracachá, Viracacha, Colombia

2 Universidad de Pamplona (Pamplona, Colombia).
}

Autor de correspondencia: Cristian Camilo Becerra Gómez. Correo electrónico: cristiancbg.cbg@gmail.com Citar este artículo así:

Becerra Gómez CC, Martínez Torres J. Tiempos y barreras de acceso para procedimientos quirúrgicos electivos en la población de Viracachá (Boyacá, Colombia), durante el periodo 2018-2019. Rev Investig Salud Univ Boyacá. 2020;7(2):65-79. https://doi.org/10.24267/23897325.566 


\title{
Waiting Times and Barriers for Elective Surgical Procedures in the Population of Vira- cacha (Boyaca, Cololmbia) during the period 2018-2019
}

\begin{abstract}
Introduction: it has been widely discussed that access to timely surgical procedures is essential in the search for population equity, always aiming for integrity in the health care system, reduction of morbimortality rates, and economic development. Waiting lists for surgical procedures have always been a huge problem for health systems due to the imbalance between patient's demand and the system's capacity to provide such procedures efficiently, mainly in rural and remote areas where financial and geographic restrictions, administrative factors, cultural beliefs, and poor education are much more present.
\end{abstract}

Objective: to determine waiting times and barriers for the performance of ambulatory surgical procedures in treated patients ESE Centro de Salud de Viracacha (Boyaca, Colombia) between 2018 and 2019.

Methodology: a following-up one cohort was performed, describing sociodemographic characteristics, elective surgery delay classification, and analysis by types of variables using person-time rates.

Results: the elective surgery was done in $48.1 \%$ of the patients with a waiting time estimated in 9,4 months $(E D=3.4)$. The operational rates per 100 months was 5.13 ( $\mathrm{Cl} 95 \%$ : 2.85-8.56). The groups who represent higher operational rates per 100 months were: patients who has socioeconomic status 1 , the group who perceived an administrative cause associated with the elective surgery delay and those who had an orthopedic surgery.

Conclusion: long waiting lists for elective surgeries may have a multifactorial component that must be identified to propose strategic policies that allow timely access to them, mainly in rural areas.

Keywords: ambulatory surgical procedures; barriers to access of health services; health services; rural population; Colombia. 


\title{
Tempos e barreiras de acesso para procedimentos cirúrgicos eletivos na população de Viraca-chá (Boyacá, Colômbia), no período 2018-2019
}

\begin{abstract}
RESUMO
Introdução: é amplamente descrito que o acesso a procedimentos cirúrgicos oportunos são essenciais para a eqüidade na atenção à saúde, bem-estar, redução da morbimortalidade e aumento do desenvol-vimento econômico. As listas de espera para cirurgias eletivas são um problema para os sistemas de saúde, devido ao desequilíbrio existente entre a demanda de pacientes e a capacidade de realizar esses procedimentos em tempo hábil, principalmente em áreas rurais, onde as restrições financeiras e geo-gráficas são muito mais acentuadas, fatores administrativos, crenças culturais e educação deficiente.
\end{abstract}

Objetivo: determinar os tempos de espera e as barreiras para procedimentos cirúrgicos eletivos em pacientes atendidos na ESE Centro de Salud de Viracachá (Boyacá, Colômbia), durante os anos de 2018 e 2019. Metodologia: estudo de acompanhamento de uma turma, com descrição sociodemográfi-ca, classificação das causas do atraso cirúrgico eletivo e análise por tipos de variáveis, usando taxas de pessoa-tempo.

Resultados: $48,1 \%$ dos pacientes realizaram o procedimento cirúrgico eletivo; em ge-ral, houve um tempo de espera de 9,4 meses ( $D P=3,4$ ) e uma taxa de operações por 100 meses de 5,13 (IC 95\%: $2,85-8,56)$. Os grupos com maiores taxas de operações por 100 meses foram os paci-entes que pertenciam ao estrato 1, aqueles que perceberam uma causa administrativa associada ao atra-so na cirurgia eletiva e aqueles que fizeram cirurgia ortopédica.

Conclusão: as longas filas de espera para cirurgias eletivas podem ter um componente multifatorial, que deve ser identificado para propor políticas estratégicas que possibilitem o acesso oportuno, principalmente, no rural.

Palavras-chave: procedimentos cirúrgicos; serviços ambulatoriais; barreiras ao acesso aos serviços de saúde; serviços de saúde; população rural; Colômbia. 


\section{INTRODUCCIÓN}

Según sostiene la Sociedad Internacional de Cirugía Ambulatoria, se define cirugía electiva o ambulatoria como una operación o procedimiento quirúrgico (excluyendo uno realizado en un consultorio), en que una persona es operada y dada de egreso el mismo día (1). Se estima que hasta el $60 \%$ de los procedimientos quirúrgicos son ambulatorios y que la selección de los pacientes se basa en el estado de salud, la complejidad de la cirugía y el sistema de recuperación y alta, con las garantías de seguridad correspondientes (2). En el mundo se ha descrito ampliamente que el acceso a procedimientos quirúrgicos oportunos es esencial en la búsqueda de equidad poblacional, para lograr la integralidad de atención en salud, bienestar, disminución de la morbimortalidad y aumento del desarrollo económico (3).

Las listas de espera de procedimientos quirúrgicos electivos son un problema para los sistemas de salud, debido al marcado desequilibrio existente entre la demanda de pacientes y la capacidad para proveerlos de manera oportuna (4). Tales factores repercuten negativamente en la calidad de vida de las personas $y$, en consecuencia, contribuyen al riesgo de deterioro sobre la salud física y mental, debido a los largos tiempos de espera hasta la realización del procedimiento.
El problema es más notorio en países como Colombia, donde no existe un modelo de priorización para tales procedimientos quirúrgicos. (5). Estudios previos han identificado que las cirugías electivas se cancelan en un $44 \%$ por motivos de tipo administrativo, mientras que el $28 \%$ se atribuyen a causas asistenciales (6). No obstante, los trámites administrativos del sistema de salud no son los únicos impedimentos para acceder de manera oportuna a una cirugía electiva; otros, como el estado de salud con necesidad de una previa optimización médica y el componente psicoemocional debido al impacto personal y social que implica someterse a una cirugía, ya se han descrito en los pacientes y se deben tener en cuenta (3).

Por consiguiente, el objetivo de este artículo fue determinar el tiempo de espera y las barreras de acceso para cirugías electivas en la población de Viracachá (Boyacá, Colombia), durante el periodo 2018-2019. Sus habitantes se tomaron como un ejemplo de población del área rural con ese tipo de dificultades, teniendo en cuenta que la complejidad de las barreras de acceso incluyen, entre otras, una evaluación de los factores médicos, sociales, administrativos y psicológicos asociados con un mayor tiempo de espera para la realización de un procedimiento quirúrgico electivo (7-9). 


\section{METODOLOGÍA}

\section{Tipo de estudio}

Este fue un estudio observacional con alcance analítico de seguimiento a una cohorte.

\section{Población y muestra}

Se ejecutó un muestreo no probabilístico por conveniencia. La población estuvo compuesta por todos los usuarios de la Empresa Social del Estado (ESE) Centro de Salud de Viracachá, quienes fueron remitidos por el servicio de consulta externa para valoración por parte de especialistas quirúrgicos entre el $1 .^{\circ}$ de enero de 2018 y el 31 de diciembre de 2019, al diagnosticarse por medicina general una probable patología que requería una cirugía electiva para su manejo. En aras de comprobar este proceso, los médicos especialistas tratantes dejan una orden por escrito, que fue consultada. Se identificaron inicialmente 76 pacientes; sin embargo, después de aplicar los criterios de exclusión, se finalizó con una muestra total de 27 pacientes.

\section{Procedimientos}

Se tuvo acceso a la base institucional de datos de remisiones ambulatorias a especialidades médico-quirúrgicas de pacientes atendidos en el centro de salud, lo que permitió identificar aquellos remitidos a una especialidad quirúrgica durante los años 2018 y 2019. Posteriormente, se revisaron las historias clínicas de dichos pacientes, mediante el software institucional, a partir de las cuales se identificaron aquellos quienes, además de ser valorados por especialistas quirúrgicos, recibieron una orden de cirugía electiva como tratamiento definitivo para su patología de base. En las historias clínicas se identificó la fecha de orden del procedimiento quirúrgico electivo, la especialidad y el nombre de la cirugía. Mediante el número telefónico inscrito en las bases de datos, el investigador principal los llamó, y así identificó e indagó en cada uno de los pacientes (de manera voluntaria y autónoma) si querían hacer parte de la investigación (consentimiento informado verbal), tiendo en cuenta el mantenimiento de la confidencialidad de los datos personales. Tras ello, les aplicó un cuestionario estructurado sobre caracterización sociodemográfica y causas de demora o no realización del procedimiento quirúrgico electivo. Se excluyeron pacientes fallecidos por complicaciones no relacionadas con la indicación quirúrgica inicial y pacientes cuyas cirugías se llevaron a cabo al ingreso por servicio de urgencias.

Se tuvieron en cuenta todos los pacientes que tenían orden médica por especialidad quirúrgica dentro del periodo comprendido entre el $1 .^{\circ}$ de enero de 2018 y el 31 de diciembre de 2019. Se realizó un seguimiento y como punto de corte 
máximo se estableció el $1 .^{\circ}$ de marzo de 2020. Esta fecha se tomó debido a la crisis sanitaria por la COVID-19, que implicó la cancelación general de todas las cirugías electivas durante las fases de importación y contingencia de la pandemia.

\section{Evaluación de la variable principal}

La variable principal del estudio fue la realización de la cirugía (sí y no) y el tiempo de espera para ella, evaluada como el número de meses desde la entrega de la orden por el especialista hasta el procedimiento quirúrgico, o, por el contrario, si no se llevó a cabo el procedimiento, hasta el punto de corte descrito previamente. Esta información se extrajo de las historias clínicas.

\section{Evaluación de variables de interés}

Para la caracterización de la población se tuvieron en cuenta las siguientes variables: sexo (masculino o femenino), edad (número de años cumplidos hasta la fecha en que se ordenó el procedimiento quirúrgico electivo), estrato socioeconómico del paciente $(1,2,3,4$ o 5), nivel de escolaridad (culminado hasta la fecha de orden de la cirugía electiva), localización geográfica (centro urbano o rural disperso) y afiliación al sistema de salud (régimen contributivo, subsidiado o régimen especial).

Finalmente, por medio de un cuestionario estructurado vía telefónica, se preguntó sobre la causa del retraso o barrera de acceso para la cirugía electiva. Se tuvieron en cuenta las siguientes variables: primero, causas administrativas (sí o no); de ser afirmativa, se definió la causa (no autorización de la entidad promotora de salud o no autorización de la institución prestadora de salud). Segundo, si hubo una causa médica que impidió o retrasó la cirugía electiva (sí o no) con el nombre de la causa médica. Por último, si existió un factor psicoemocional implicado directamente con el retraso o no realización del procedimiento quirúrgico electivo (sí o no).

\section{Análisis estadístico}

Inicialmente, se desarrolló un análisis exploratorio $y$, posterior a esto, se describieron las variables de interés por sexo. Las variables cualitativas se reportaron por medio de frecuencias porcentuales y frecuencias absolutas. Entre tanto, las variables cuantitativas que presentaban dispersión simétrica se describieron a través de la media y desviación estándar, y en caso contrario, se expresaron con la mediana y cuartil 1 y 3.

Para evaluar el tiempo de espera para la realización de la cirugía en cada una de las variables de interés, se describió la cantidad, la frecuencia porcentual y la sumatoria de meses en cada una de las categorías de las variables estudiadas; adicionalmente, se calculó la tasa de operaciones en cada persona/ mes y se estimó el intervalo de confianza con el 
método del Test Exacto Mid-P. Finalmente, se contrastaron las tasas entre las mayores y las menores de cada una de las categorías de variables, por medio del estimador de máxima verosimilitud de la razón de tasas (CMLE) y se reportaron los intervalos de confianza al $95 \%$.

Las tasas y las razones de tasas se analizaron mediante el ingreso de datos a la página web https://www.openepi.com, con herramientas estadísticas epidemiológicas de código abierto para salud pública. Los datos se procesaron por medio del formato de recolección de información a través de página web SURVEYWIKI y directamente de las historias clínicas de los pacientes objeto de estudio. Posteriormente, estos se exportaron a Microsoft Excel, donde se consolidó una base de datos para su análisis estadístico por medio del software SPSS Statistics Base 22.0.

\section{Declaración de sesgos}

Selección: a pesar de que se intentó incluir a toda la población que cumpliera con los criterios de selección, es probable que durante los años 2018 y 2019 hubieran faltado los datos de algunos de los pacientes en la base de datos de solicitudes para valoración por especialistas. Ello generaría la exclusión de personas que hacen parte de la población seleccionada (10-12).
Clasificación: con respecto a las variables extraídas de las historias clínicas, es importante declarar que pueden tener imprecisiones, debido a que no son parte de procesos de investigación, sino de completitud de formatos en salud. Por otro lado, con respecto a las variables evaluadas por la encuesta estructurada, puede incurrirse en un sesgo de medición en la que el paciente no recuerde el motivo de demora del procedimiento quirúrgico electivo; adicionalmente, esas características se evaluaron mediante un autorreporte, que no se encuentra validado para tal fin. Por último, todas las variables se valoraron en el momento en que se desarrolló la orden médica y no se tuvieron en cuenta factores que se pueden modificar con el tiempo $(10,13)$.

\section{Aspectos éticos}

La aprobación de este estudio fue avalada por el Comité de Ética Médica de la ESE Centro de Salud de Viracachá, Boyacá. Se clasificó el estudio como de riesgo mínimo, según los principios establecidos en la Declaración de Helsinki y en la Resolución 008430 del 4 de octubre de 1993. Adicionalmente, todos los pacientes que participaron en el estudio dieron su consentimiento informado de manera verbal, mediante llamada telefónica. Así se respetó su autonomía y se guardó la confidencialidad de sus datos personales. 


\section{RESULTADOS}

Inicialmente, se identificaron 76 pacientes que durante los años 2018 y 2019 fueron remitidos de manera ambulatoria y valorados por médico especialista del área quirúrgica, y a quienes se les entregó una orden para un procedimiento quirúrgico indicado por su patología de base. De ellos, 30 tenían datos incompletos o no quisieron participar en el estudio. Adicionalmente, 8 pacientes, pese al diagnóstico de patología con indicación quirúrgica, decidieron de manera autónoma no realizarse el procedimiento quirúrgico electivo. Además, 3 pacientes fallecieron por causa médica no relacionada con la patología quirúrgica. Finalmente, después de aplicar todos los criterios de inclusión y exclusión, la muestra final fue de 27 pacientes que fueron valorados por médico especialista y a quienes se les ordenó una cirugía electiva.

De los 27 sujetos estudiados, se encontró un total de 12 hombres $(44,4 \%)$, en edades desde los 8 hasta los 74 años. El promedio de edad para los hombres fue de 57,5 años; mientras que para las mujeres correspondió a los 50,6 años. Las cirugías más ordenadas fueron: herniorrafía inguinal abierta $(29,6 \%)$, histerectomía abdominal total $(18,5 \%)$ y remplazo total de cadera $(11,1 \%)$. Las otras cirugías corresponden al 59,2\%. En relación con las barreras de acceso para la no ejecución o demora del procedimiento quirúrgico electivo, se encontró que las causas administrativas corresponden al 76,9\%; en tanto que las causas médicas y psicosociales equivalen al $11,1 \%$ cada una. El resto de las variables sociodemográficas se analizan en la tabla 1.

Se encontró que al 48,1 \% de los pacientes habían recibido el procedimiento quirúrgico electivo, con un tiempo de espera de 9,4 meses $(D E=3,4 ;$ ) y una tasa de operaciones por cada 100 meses de 5,13 (IC95\%: 2,85-8,56). Los grupos que presentaron mayores tasas de operaciones por cada 100 meses fueron: pacientes que pertenecían al estrato 1 (7,69; IC95\%: 3,75-14,12), aquellos tuvieron una cirugía ortopédica $(7,69$; IC95\%: 3,1116) y los que percibieron tener causas administrativas como retraso del procedimiento quirúrgico $(6,25$; IC95\%: 3,38-10,63) y los del sexo masculino $(6,06 ;$ IC95\%: 2,81-11,51).

Al estimar las razones de las tasas de operaciones, se encontró que los dos últimos grupos tuvieron un resultado de mayor magnitud comparado con el grupos de referencia: $(2,84$; IC95\%: 0,42$65,92)$ y $(2,56$; IC95\%: 0,44-55,29), respectivamente. Sin embargo, ninguna las variables mostraron una asociación significativa. Los demás datos del tiempo de espera para la realización de la cirugía, tasa de operaciones por cada 100 meses y las razones de las tasas de operaciones se describen en la tabla 2. 
Tabla 1. Descripción sociodemográfica de los pacientes a los que les ordenaron cirugía electiva en la ESE Centro de salud de Viracachá, 2018-2019

\begin{tabular}{|c|c|c|c|c|c|c|}
\hline \multirow{3}{*}{ Variable } & \multirow{2}{*}{\multicolumn{2}{|c|}{$\begin{array}{l}\text { Hombre } \\
(\mathrm{n}=12) \\
\end{array}$}} & \multirow{2}{*}{\multicolumn{2}{|c|}{$\begin{array}{c}\text { Mujer } \\
(\mathrm{n}=15) \\
\end{array}$}} & \multirow{2}{*}{\multicolumn{2}{|c|}{$\begin{array}{c}\text { Total } \\
(\mathbf{n}=\mathbf{2 7}) \\
\end{array}$}} \\
\hline & & & & & & \\
\hline & & DE & $\bar{x}$ & $\mathrm{DE}$ & $\bar{x}$ & DE \\
\hline Tiempos de espera (meses) & 11 & 2,9 & 8,1 & 3,9 & 9,4 & 3,7 \\
\hline Realización del procedimiento quirúrgico electivo & $\mathrm{n}$ & $\%$ & $\mathrm{n}$ & $\%$ & $\mathrm{n}$ & $\%$ \\
\hline Sí & 8 & 66,7 & 5 & 33,3 & 13 & 48,1 \\
\hline No & 4 & 33,3 & 10 & 66,7 & 14 & 51,9 \\
\hline \multicolumn{7}{|l|}{ Edades agrupadas } \\
\hline Adolescentes-adulto joven & 1 & 8,3 & 2 & 13,3 & 3 & 11,1 \\
\hline Adultez intermedia & 5 & 41,7 & 10 & 66,7 & 15 & 55,6 \\
\hline Adultez tardía & 6 & 50 & 3 & 20 & 9 & 33,3 \\
\hline \multicolumn{7}{|l|}{ Estrato socioeconómico } \\
\hline Estrato 1 & 9 & 75 & 9 & 60 & 18 & 66,7 \\
\hline Estrato 2 & 3 & 25 & 6 & 40 & 9 & 33,3 \\
\hline \multicolumn{7}{|l|}{ Localización geográfica } \\
\hline Centro urbano & 1 & 8,3 & 4 & 26,6 & 5 & 18,5 \\
\hline Rural disperso & 11 & 91,7 & 11 & 73,4 & 22 & 81,5 \\
\hline \multicolumn{7}{|l|}{ Nivel educativo } \\
\hline Terminaron primaria básica & 3 & 25 & 13 & 86,7 & 16 & 59,2 \\
\hline No terminaron primaria básica & 9 & 75 & 2 & 13,3 & 11 & 40,8 \\
\hline \multicolumn{7}{|l|}{ Especialidad que indicó cirugía electiva } \\
\hline Cirugía general & 6 & 50 & 2 & 13,3 & 8 & 29,7 \\
\hline Ginecología & 0 & 0 & 6 & 40 & 6 & 22,2 \\
\hline Urología & 1 & 8,3 & 2 & 13,3 & 3 & 11,1 \\
\hline Ortopedia & 5 & 41,7 & 2 & 13,3 & 7 & 25,9 \\
\hline Otras especialidades quirúrgicas & 0 & 0 & 3 & 20,1 & 3 & 11,1 \\
\hline \multicolumn{7}{|c|}{ Causa administrativa impidió realización oportuna de cirugía electiva* } \\
\hline Sí & 8 & 72,7 & 12 & 80 & 20 & 76,9 \\
\hline No & 3 & 27,3 & 3 & 20 & 6 & 23,1 \\
\hline \multicolumn{7}{|c|}{ Causa médica impidió realización oportuna de cirugía electiva } \\
\hline Sí & 2 & 16,7 & 1 & 6,7 & 3 & 11,1 \\
\hline No & 10 & 83,3 & 14 & 93,3 & 24 & 88,9 \\
\hline \multicolumn{7}{|c|}{ Motivos psicoemocionales impidieron realización oportuna de cirugía electiva } \\
\hline Sí & 1 & 8,3 & 2 & 13,3 & 3 & 11,1 \\
\hline No & 11 & 91,7 & 13 & 86,7 & 24 & 88,9 \\
\hline
\end{tabular}

n: número de pacientes; promedio; DE: desviación estándar. * Un dato perdido. 
Tabla 2. Asociación entre el tiempo de espera para la cirugía electiva en los pacientes de la ESE Centro de salud de Viracachá, 2018 y 2019

\begin{tabular}{|c|c|c|c|c|c|}
\hline & \multicolumn{2}{|c|}{$\begin{array}{c}\text { Realización de } \\
\text { operaciones }^{A}\end{array}$} & \multirow[t]{2}{*}{$\begin{array}{c}\text { Cantidad de } \\
\text { meses }^{\mathrm{B}}\end{array}$} & \multirow{2}{*}{$\begin{array}{c}\begin{array}{c}\text { Tasa operaciones } \\
\text { persona-meses }^{\mathrm{C}}\end{array} \\
(\mathrm{IC} 95 \%)\end{array}$} & \multirow{2}{*}{$\begin{array}{c}\begin{array}{c}\text { Razón tasa operaciones } \\
\text { persona-meses }\end{array} \\
\text { (IC95\%) }\end{array}$} \\
\hline & $\mathbf{n}$ & $\%$ & & & \\
\hline Total $(\mathrm{n}=27)$ & 13 & 48,2 & 253 & $5,13(2,85-8,56)$ & No aplica \\
\hline \multicolumn{6}{|l|}{ Sexo } \\
\hline Masculino $(\mathrm{n}=12)$ & 8 & 66,6 & 132 & $6,06(2,81-11,51)$ & $1,46(0,47-4,92)$ \\
\hline Femenino $(\mathrm{n}=15)$ & 5 & 33,3 & 121 & $4,13(1,51-9,15)$ & REF \\
\hline \multicolumn{6}{|l|}{ Edad agrupada } \\
\hline $\begin{array}{l}\text { Adolescente-adulto joven ( } \mathrm{n} \\
=3 \text { ) }\end{array}$ & 2 & 66,6 & 39 & $5,12(0,85-16,94)$ & $1,11(0,15-5,66)$ \\
\hline Adultez intermedia $(\mathrm{n}=15)$ & 5 & 33,3 & 109 & $4,58(1,68-10,17)$ & REF \\
\hline Adultez tardía $(\mathrm{n}=9)$ & 6 & 66,6 & 105 & $5,71(2,31-11,89)$ & $1,24(0,36-4,43)$ \\
\hline \multicolumn{6}{|l|}{ Estrato socioeconómico } \\
\hline Estrato $1(\mathrm{n}=18)$ & 9 & 50,0 & 177 & $7,69(3,75-14,12)$ & $0,96(0,32-3,6)$ \\
\hline Estrato $2(n=9)$ & 4 & 44,4 & 76 & $5,26(1,67-12,7)$ & REF \\
\hline \multicolumn{6}{|l|}{ Localización geográfica } \\
\hline Centro urbano $(n=5)$ & 1 & 20,0 & 32 & $3,12(0,15-15,41)$ & REF \\
\hline Rural disperso $(\mathrm{n}=22)$ & 12 & 44,4 & 221 & $5,43(2,94-9,23)$ & $1,73(0,30-37,4)$ \\
\hline \multicolumn{6}{|l|}{ Nivel educativo } \\
\hline $\begin{array}{l}\text { Terminaron primaria básica } \\
(\mathrm{n}=16)\end{array}$ & 6 & 37,5 & 132 & $4,54(1,84-9,54)$ & $\mathrm{REF}$ \\
\hline $\begin{array}{l}\text { No terminaron primaria básica } \\
(\mathrm{n}=11)\end{array}$ & 7 & 63,6 & 118 & $5,93(2,59-11,73)$ & $1,30(0,42-4,13)$ \\
\hline \multicolumn{6}{|c|}{ Especialidad que indicó cirugía electiva ${ }^{\mathrm{D}}$} \\
\hline Cirugía general $(\mathrm{n}=8)$ & 4 & 50,0 & 74 & $5,40(1,71-13,04)$ & $2(0,25-49,49)$ \\
\hline Ginecología $(n=6)$ & 2 & 33,3 & 43 & $4,65(0,77-15,37)$ & $1,72(0,13-50,76)$ \\
\hline Urología $(n=3)$ & 1 & 33,3 & 37 & $2,70(0,13-13,33)$ & REF \\
\hline Ortopedia $(n=7)$ & 6 & 85,7 & 78 & $7,69(3,11-16)$ & $2,84(0,42-65,92)$ \\
\hline \multicolumn{6}{|c|}{ Causa administrativa no permitió realización oportuna de procedimiento quirúrgico } \\
\hline Sí $(\mathrm{n}=21)$ & 12 & 57,1 & 192 & $6,25(3,38-10,63)$ & $2,56(0,44-55,29)$ \\
\hline No $(n=6)$ & 1 & 16,6 & 41 & $2,43(0,12-12,03)$ & REF \\
\hline \multicolumn{6}{|c|}{ Causa médica impidió realización oportuna de cirugía electiva } \\
\hline Sí $(\mathrm{n}=3)$ & 1 & 33,3 & 27 & $3,70(0,18-18,27)$ & REF \\
\hline No $(\mathrm{n}=24)$ & 12 & 50,0 & 226 & $5,31(2,87-9,02)$ & $1,43(0,24-40,93)$ \\
\hline
\end{tabular}




\begin{tabular}{lccccc}
\hline \multicolumn{5}{c}{ Motivos psico-emocionales que impidieron realización oportuna de cirugía electiva } \\
\hline Sí $(\mathrm{n}=3)$ & 0 & 0 & 14 & 0 & No aplica \\
\hline No $(\mathrm{n}=24)$ & 13 & 54,2 & 239 & $5,43(3,01-9,06)$ & no aplica \\
\hline
\end{tabular}

$\mathrm{n}$ : Número de pacientes; $\mathrm{A}$ : frecuencia absoluta y acumulada de pacientes que se realizaron la cirugía en cada categoría de cada variable; B: sumatoria de meses de espera para la realización de la cirugía en cada categoría de cada variable; C: cociente entre la cantidad de cirugía y la sumatoria de meses de espera para la realización de la cirugía en cada categoría de cada variable, dicha tasa se expresa por 100 meses; D: se excluyeron las personas que tenían orden por otras especialidades quirúrgicas; IC95\%: intervalo de confianza del 95\%; REF: grupo de referencia para la comparación de las tasas.

\section{DISCUSIÓN}

En el presente estudio se identificaron las posibles barreras de acceso y las demoras en la realización de los procedimientos quirúrgicos electivos en la población del municipio de Viracachá (Boyacá), basados en las políticas de cirugía global de la Organización Mundial de la Salud, los cuales describen las dificultades para el acceso oportuno de procedimientos quirúrgicos, especialmente en países en vías de desarrollo y en áreas rurales, donde son mucho más marcadas las restricciones financieras y geográficas, los factores administrativos, las creencias culturales y la pobre educación $(3,14)$.

Se estima que en todo el mundo se llevan a cabo 234,2 millones de cirugías mayores cada año y que el $30 \%$ de la población mundial se somete al $73,6 \%$ de esos procedimientos. Se resalta que el tercio más pobre de la población solamente recibe el $3,5 \%$ de dichos procedimientos $(14,15)$. De esa manera se demuestra la enorme brecha de acceso quirúrgico en países en vías de desarrollo.
Un estudio de cohortes publicado en 2020 por Alty et al. (16) identificó las barreras de acceso quirúrgico en el área rural de Burundi (África oriental), donde se encontraron factores asociados al retraso de acceso quirúrgico. Allí se destacó la edad avanzada (HR: 0,99; IC95\%: 0,98-0,99) y usos de curandero tradicional (HR: 0.528; IC95\%: 0,31-0,90). Por otro lado, los factores asociados con la disminución del retraso en los problemas quirúrgicos se identificaron con el mayor nivel educativo (HR: 1,06; IC95\%: 1,004-1,11). Nuestro estudio identificó como principales características sociodemográficas, para el retraso de procedimientos quirúrgicos electivos, estar en el grupo de los hombres, edades de adultez tardía (mayores de 65 años), cirugías indicadas por especialistas en ortopedia y cirugía general, estratos socioeconómicos 1 y 2 y personas con más bajo nivel educativo y con vivienda en el área rural dispersa.

En nuestro estudio, las causas administrativas fueron percibidas como el principal factor del 
tiempo de demora en la realización, en un $77,7 \%$. Comparado con un estudio de Gaviria-García et al. (6), en Barranquilla (Colombia), se encontró que la perspectiva de demoras administrativas en un $33 \%$ se debe al personal de salud. Nuestro estudio indica que de todas las causas administrativas, la falta de autorización por parte de las empresas promotoras de salud es las más frecuente, con un $95,2 \%$.

Las barreras culturales también pueden estar implicadas en la dificultad para la atención quirúrgica oportuna; entre ellas se han destacado: el miedo a la anestesia y el miedo a los malos desenlaces como consecuencia de la realización de una cirugía (14).

Un estudio publicado por Chandrashekhar et al. (17), en India, encontró que el 19,2\% de pacientes con cataratas e indicación quirúrgica de faquectomía tenía miedo a la cirugía. Nuestro estudio encontró que, de la muestra analizada, incluyendo todas las cirugías, el $11,5 \%$ de los pacientes no permitió el procedimiento quirúrgico por miedo o ansiedad frente a la cirugía.

Las causas médicas, por otro lado, también han sido un determinante para el retraso de un procedimiento quirúrgico electivo; pero no son vistas siempre desde un aspecto negativo, más aún con el progresivo surgimiento de políticas institucionales de medicina perioperatoria y protocolos del tipo Enhanced Recovery After Surgery (ERAS), que buscan la optimización de un paciente antes de ser llevado a una cirugía con el fin de alcanzar desenlaces favorables respecto a morbimortalidad de pacientes quirúrgicos (18). En nuestros resultados encontramos que el $11,5 \%$ de los pacientes tuvieron una causa médica como retraso en su cirugía electiva indicada por especialista. Se destaca que el $66,6 \%$ fue por causa de patologías de origen cardiaco, y el restante 33,3\% correspondió a enfermedades de otras etiologías.

La principal limitación metodológica de nuestro estudio implica la dificultad para hacer asociaciones estadísticamente significativas $y$, en consecuencia, al tipo de estudio y la muestra reducida de pacientes, que, además, podría implicar la presencia de factores causantes de confusión que no se incluyeron en este estudio y que durante el análisis de resultados pueden haber tenido una multicausalidad, debido a la compleja dinámica social, económica y personal que pudiese existir en un mismo resultado.

La identificación de factores asociados al retraso o no realización de procedimientos quirúrgicos electivos puede servir como base para plantear políticas estratégicas que disminuyan la brecha de inequidad para una atención integral y el acceso oportuno de cirugías electivas en la población en general; pero principalmente en áreas rurales y de dificultad de acceso a servicios de 
salud, donde existe un mayor número de limitaciones que pueden repercutir negativamente en la salud física y mental de las personas.

\section{CONCLUSIONES}

En la población de pacientes del municipio de Viracachá, Boyacá, durante los años 2018-2019, se encontró un mayor tiempo de espera para cirugías electivas en hombres, pacientes de edad avanzada, estratos bajos, indicadas por ortopedia y cirugía general, vivienda en área rural dispersa y tener un bajo nivel educativo. La principal razón de la mayor demora fue percibida por causas administrativas, seguidas de causas médicas y psicosociales.

Teniendo en cuenta lo anterior, se pretende que nuestro estudio sirva como una base metodológica e investigativa en Colombia para el desarrollo de estudios más grandes que permitan obtener resultados a una mayor escala y que representen las necesidades poblacionales, partiendo de la premisa de que la atención quirúrgica es un componente integral de los sistemas de salud para los países en todos los niveles de desarrollo (19).

\section{AGRADECIMIENTOS}

El autor principal agradece a todos los participantes incluidos en este estudio y a las directivas de la ESE Centro de Salud de Viracachá, Boyacá, por el apoyo brindado.

\section{CONFLICTO DE INTERESES}

Los autores declaramos no tener ningún conflicto de intereses.

\section{FINANCIACIÓN}

El estudio tuvo el apoyo de los directivos de la ESE Centro de Salud de Viracachá (Boyacá, Colombia).

\section{REFERENCIAS}

1. International Association for Ambulatory Surgery. Ambulatory (day) surgery: suggested international terminology and definitions [internet]. París; 2003. Disponible en: https:// www.iaas-med.com/files/historical/IAAS definitions.pdf

2. Valente R, Testi A, Tanfani E, Fato M, Porro I, Santo $M$, et al. A model to prioritize access to elective surgery on the basis of clinical urgency and waiting time. BMC Health Serv Res. 2009;9(1). https://doi.org/10.1186/1472-6963-9-1

3. Meara J, Greenberg S. Hagander L, Alkire BC, Alonso N, Ameh EA, et al. The Lancet Commission on Global Surgery Global surgery 2030: evidence and solutions for achieving health, welfare and economic development. Surgery. 2015;386(9963):569-624. https://doi. org/10.1016/S0140-6736(15)60160-X 
4. Curtis AJ, Russell $\mathrm{COH}$, Stoelwinder JU, McNeil $\mathrm{JJ}$. Waiting lists and elective surgery: ordering the queue. Med J Austr. 2010;192(4):217-20. https:// doi.org/10.5694/j.1326-5377.2010.tb03482.x

5. Oudhoff J, Timmermans D, Knol D, Bijnen A, van der Wal G. Waiting for elective general surgery: impact on health related quality of life and psychosocial consequences. BMC Public Health. 2007;7(164). https://doi. org/10.1186/1471-2458-7-164

6. Gaviria-García G, Lastre-Amell G, Suárez-Villa M. Causas que inciden en cancelación de cirugías desde la percepción del personal de salud. Enferm Univ [internet]. 2014;11(2):4751. Disponible en: http://www.scielo.org.mx/ pdf/eu/v11n2/v11n2a2.pdf

7. Hilkhuysen $G$, Oudhoff J, Rietberg M, van der Wal G, Timmermans D. Waiting for elective surgery: a qualitative analysis and conceptual framework of the consequences of delay. Public Health. 2005;119(4):290-3. https://doi. org/10.1016/j.puhe.2004.05.020

8. Departamento Administrativo Nacional de Estadísticas. Resultados del Censo Nacional de Población y Vivienda 2018 [internet]. Disponible en: https://www.dane.gov.co/index.php/estadisticas-por-tema/demografia-y-poblacion/ censo-nacional-de-poblacion-y-vivenda-2018
9. Rojas Ayala R. E.S.E Centro de Salud de Viracachá: análisis de situación de salud con el modelo de los determinantes sociales de salud, municipio de Viracachá Boyacá [internet]. Tunja: Secretaría de Salud de Boyacá; 2018. Disponible en: https://www.boyaca.gov. co/SecSalud/images/Documentos/Asis2018/ asis_\%20viracacha_2018.pdf

10. Delgado-Rodríguez M, Llorca J. Bias. J Epidemiol Community Health. 2004;58(8):635-41. http://doi.org/10.1136/jech.2003.008466

11. Hernán MA, Hernández-Díaz S, Robins JM. A structural approach to selection bias. Epidemiology. 2004;15(5):615-25. http://doi. org/10.1097/01.ede.0000135174.63482.43

12. Ellenberg JH. Selection bias in observational and experimental studies. Stat Med. 1994;13:55767. http://doi.org/10.1002/sim.4780130518

13. Höfler M. The effect of misclassification on the estimation of association: a review. Int J Methods Psychiatr Res. 2005;14(2):92-101. https://doi.org/10.1002/mpr.20

14. Grimes $C E$, Bowman $K G$, Dodgion CM, Lavy CB. Systematic review of barriers to surgical care in low-income and middle-income countries. World J Surg. 2011;35:941-50. https:// doi.org/10.1007/s00268-011-1010-1 
15. Weiser TG, Regenbogen SE, Thompson KD, Haynes AB, Lipsitz SR, Berry WR, et al. An estimation of the global volume of surgery: a modelling strategy based on available data. Lancet. 2008;372(9633):139-44. https://doi. org/10.1016/S0140-6736(08)60878-8

16. Alty IG, Niyukuri A, Niyuhire M, Dee EC, Jhon T, Fader JP, et al. Sociodemographic, barriers to surgical care access in rural burundi: sociodemographic, transportation, and care-seeking patterns associated with delay in access to surgical care. J Am Chem Soc. 2020;231(4):S119-20. https://doi.org/10.1016/j.jamcollsurg.2020.07.229

17. Chandrashekhar TS, Bhat HV, Pai RP, Nair SK. Coverage, utilization and barriers to cataract surgical services in rural South India: results from a population-based study. Public Health. 2007;121(2):130-6. https://doi.org/10.1016/j. puhe.2006.07.027

18. Aronson S, Martin G, Gulur P, Lipkin ME, Lagoo-Deenadayalan SA, Mantyh CR, et al. Properative optimization: a continued call of action. Anesth Analg. 2020;130(4):808-10. https:// doi.org/10.1213/ANE.0000000000004492
19. Meara JG, Hagander L, Leather AJ. Surgery and global health: a Lancet Commission. Lancet. 2014;383(9911):12-3. https://doi. org/10.1016/S0140-6736(13)62345-4

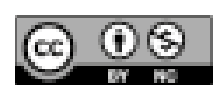

Esta obra está bajo una licencia de Creative Commons Reconocimiento-NoComercial 4.0 Internacional 